Paper

\title{
Object Manipulation for Perceiving a Sense of Material using User-Perspective Mobile Augmented Reality
}

\author{
Ryota Nomura $^{\dagger}$, Takashi Komuro (member) ${ }^{\dagger}$, Shoji Yamamoto (member) ${ }^{\dagger \dagger}$, \\ Norimichi Tsumura (member) ${ }^{\dagger \dagger \dagger}$
}

\begin{abstract}
In this study, we conducted an experiment using a mobile augmented reality (AR) system that allows direct manipulation of a virtual object with the user's hand and that presents material appearance of the object. The system presents user-perspective images on the mobile display, which enables natural interaction with virtual objects. We compared the methods with user-perspective and camera-perspective presentation using an object manipulation task for material perception. The result showed that the answer time in the task became significantly shorter with user-perspective presentation, which indicates that user-perspective presentation makes manipulation of virtual objects in mobile AR easier.
\end{abstract}

Key words: Mobile augmented reality, bare hand interaction, 3D Manipulation, material appeararnce.

\section{Introduction}

In Internet shopping, it is important to present material appearance of products for enhancing consumers' willingness of purchasing the products. Material appearance of objects is largely affected by their reflectance. To present realistic material appearance, it is necessary to allow users to see the objects from different directions to observe reflection change on their surfaces. To make users perceive a strong sense of material, it would be effective to allow them to manipulate objects using their hand which enables intuitive 3D operation.

There have been augmented reality (AR) systems using mobile displays that allow direct interaction with virtual objects using users' hand ${ }^{1)^{\sim}} 7$. However, cameraperspective images are presented on the display and the hand in the images is different in position and size from the real hand. This may cause inconsistency between the users' visual and somatosensory perception and give the users strange feeling.

On the other hand, AR systems that allow direct interaction with virtual objects using head-mounted displays (HMDs) have been proposed..$^{8{ }^{\sim 12}}$. HMDs can present user-perspective images to users, which enables

\footnotetext{
Received Aprir 22, 2020; Revised June 9, 2020; Accepted June 17, 2020

$\dagger$ Saitama University

(Saitama, Japan)

$\dagger †$ Tokyo Metropolitan College of Industrial Technology

(Tokyo, Japan)

†† Chiba University

(Chiba, Japan)
}

more natural interaction with virtual objects. However, HMDs are not yet widely widespread to consumers and it may be difficult for users to newly introduce such devices.

To solve the problem of view inconsistency in mobile AR, there have been studies that present userperspective images on mobile displays ${ }^{13)^{\sim 22}}$. It is expected that user-perspective presentation can resolve the inconsistensy between the user's vision and somatosensory perception and enables more natural interaction with virtual objects in mobile AR.

Based on these studies, we have proposed a mobile AR system that enables direct manipulation of virtual objects using the user's hand and that presents the material appearance of the objects ${ }^{23)}$. The system presents user-perspective images on the mobile display to realize natural interaction with virtual objects, and allows the user to move and rotate a virtual object with six degrees of freedom (three degrees of freedom for translation and three degrees of freedom for rotation) by mapping the hand pose to the object's translation and rotation.

In this study, we conducted an experiment using our system to verify if user-perspective presentation is effective for object manipulation and enhances the material perception. We believe that not only visual information but also physical actions such as moving an object in one's hand is important for material perception, and the matching of visual and somatosensory information is expected to enhance the material perception. We compared the methods with user-perspective and cameraperspective presentation using an object manipulation 


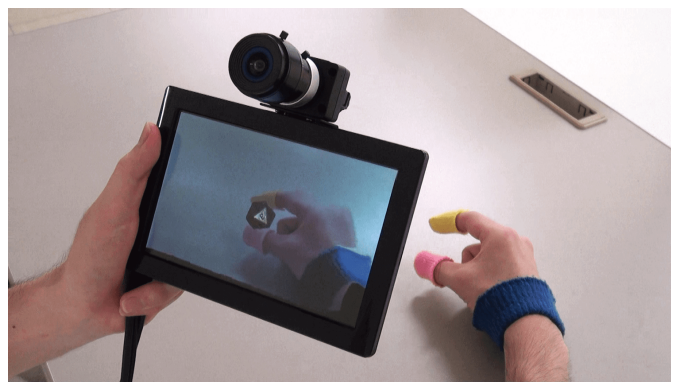

Fig. 1 A user manipulating a virtual object

task for material perception.

\section{Mobile AR System for Presenting Ma- terial Appearance}

Using our system, a user can manipulate a virtual object with six degrees of freedom in real time by moving the user's hand and fingers on which color markers are put. The user can obtain the gloss and burnish on the surfaces of the object and perceive a sense of the material.

Our system uses the same hardware configuration as the see-through mobile AR system proposed $i^{19)}$. This system displays images of real scenes on the mobile display so that the appearance of the images on the screen becomes consistent with that in the real space from the user's viewpoint position. The system consists of a mobile display, a camera that is attached in front of a mobile display, and a depth camera that is attached to the rear center of the mobile display. The system obtains the 3D scene behind the display using the depth camera, and the user's viewpoint position by face tracking using the front camera. Then, the system generates user-perspective images by reprojecting the $3 \mathrm{D}$ scene on the display according to the user's viewpoint position.

To realize object manipulation, the system detects the regions of the color markers on the hand from the color image, and converts the $2 \mathrm{D}$ coordinates of the pixels in each region into 3D coordinates using the depth image and calculates their centroid. Using the centroids of all markers, the user's hand pose is calculated and is mapped to the object's translation and rotation.

Figure 1 shows a user manipulating a virtual object using our system. The user is holding the device with one hand, and manipulating a virtual object using the other hand on which three color markers are put.

\section{Experiment}

We conducted an experiment to verify if userperspective presentation is effective for object manipu-

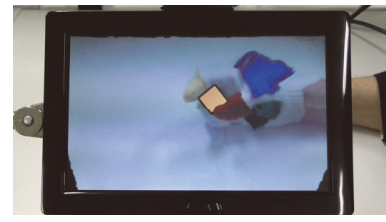

(a) user-perspective

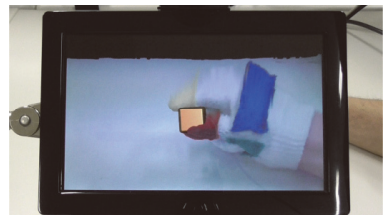

(b) camera-perspective
Fig. 2 Appearance of each method

lation and enhances the material perception. We asked the participants to perform a task to distinguish between different materials of objects, and measured correct answer rates and answer time. We used correct answer rates as an indicator to evaluate the level of material perception since high correct answer rates indicate that the participants were able to accurately distinguish between different materials. We used answer time as an indicator to evaluate the operability of the system since it is strongly influenced by the operability rather than material perception.

\section{1 Methods}

We compared two methods. One is object manipulation with user-perspective presentation (userperspective) and the other is that with cameraperspective presentation (camera-perspective).

In user-perspective, if we set the viewpoint to the user's dominant eye and the user puts his/her hand to a natural position on the screen, some of the markers on the hand are out of the view of the depth camera. Therefore, we set the viewpoint to the midpoint of the user's both eyes. In addition, to stabilize images displayed on the screen, we set the system not to change the viewpoint until it moves $8 \mathrm{~cm}$ from the previous position, which is about half the typical face width and that was empirically determined.

In camera-perspective, we fixed the viewpoint to the position that was $8.4 \mathrm{~cm}$ above the center of the display, which was determined from the vertical distance between the center of the screen and the rear camera of a 9.7 inch tablet device such as iPad when a user hold it horizontally. We set the angle of view to 35 degree so that a virtual object looks as large as that in user-perspective and also the black region in displayed images, which corresponds to the out-of-view region of the depth camera, becomes as small as possible.

To improve the manipulation accuracy, we asked the participants to wear a white glove with five color markers put on the thumb, index finger, middle finger, palm, and back when manipulating a virtual object. Figure 2 the appearance of each method. 


\section{2 Design}

\section{(1) Participants}

We recruited twelve student volunteers (4 female, 8 male) whose ages ranged from 20 to 28 (mean: 23.0, SD: 1.8). We paid 3,600 JPY (about 32 USD) to each participant. We restricted the participants to righthanded persons since our system sometimes fails to obtain 3D positions of the markers when manipulation is performed with a left hand, which is due to the difference in positions of the sensors for color and depth images. All the participants had normal vision. Nine knew what $A R$ is and had experience of $A R$, and the others only knew the name. Six often played computer games, five sometimes did, and one never did.

\section{(2) Environment}

Each participant was seated in a chair, wore a glove with markers on his/her right hand, and performed direct manipulation of virtual objects using the hand. We fixed the experimental device above a desk using a stand, and adjusted the height and orientation of the device according to the pose of the seated participant. The participant put a keyboard on his/her lap and operated it with the left hand.

Figure 3 (a) shows a photograph of a user performing the task and Fig. 3 (b) shows the schematic diagram of the geomertic relationship. The geometric relationship among the user's eye, hand, and the display is common in user-perspective and camera-perspective, but the fields of view of the virtual cameras are different.

\section{(3) Task}

In the task, we used three virtual objects (Object A, Object B, Reference), which were cube-shaped with the edges rounded off. Each of three objects had five faces with the same material (color and reflectance) and one face with a difference material. The material for the five faces was designed to be matte and that for the one face was designed to be glossy so that highlight can be observed only on the one face. The reason why we used a different material for only one face was that there are often situations where we want to know the material appearance of a part of an object in real applications. We also wanted to unify the way the participants manipulate objects by limiting it to such situations.

The reflectance parameters for the five faces with the same material were common among three objects, but the parameters for the face with different material were common only between either of Object A or Object B (randomly chosen) and Reference, and a certain variation was added to a parameter of the other object.

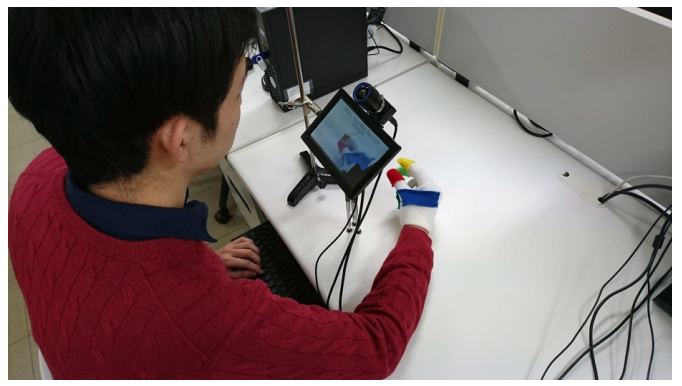

(a)

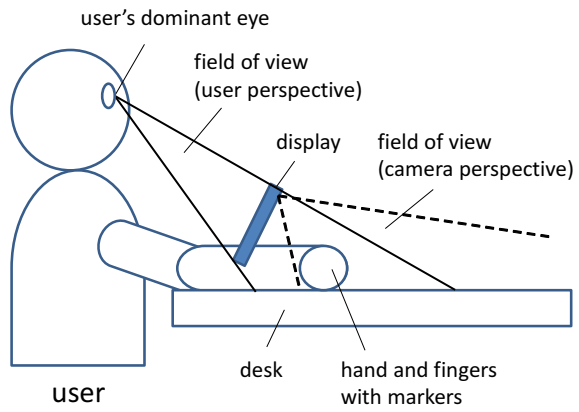

(b)

Fig. 3 Experimental environment: (a) a user performing the task, (b) geometric relationships.

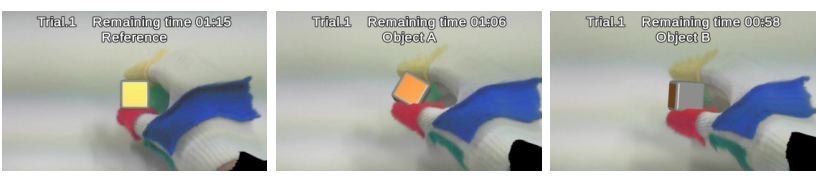

Fig. 4 Object Manipulation Task for Perceiving a Sense of Material

That is, either of Object A or B and Reference had completely the same reflectance, but the other object had slightly different reflectance in the specific face.

If we use objects with curved faces such as roundshaped objects, the difference in reflectance can be easily distinguished from the appearance of highlight, and also the appearance of highlight does not largely change even when the objects are rotated. On the other hand, if we use objects with sharp edges, the appearance of highlight around edges suddenly changes when the objects are rotated, which makes it easy to distinguish the difference. That is why we used cube-shaped objects with the edges rounded off.

The task was to manipulate these objects, to observe the appearance change, and to answer which of Object A and Object B has different material appearance from that of Reference in a time limit. Figure 4 shows screen images while a participant is performing the task. The current trial number, remaining time, and the name of the object are shown at the top of the screen.

One of the three virtual objects was displayed on the 
screen at a time, and the participant was able to switch the object to the next one by pressing the space key. When the object was switched, the current object disappeared and no object was shown for two seconds. After that, the next object appeared with a random pose. The object was switched in the order of Reference $\rightarrow$ Object $\mathrm{A} \rightarrow$ Object $\mathrm{B} \rightarrow$ Reference $\rightarrow \cdots$. The participant was able to switch the object any number of times within the time limit. After comparing three objects enough, the participant answered the object that had different material appearance from that of Reference by pressing ' $\mathrm{V}$ ' key (Object A) or 'B' key (Object B). This trial was repeated 15 times. In each trial, no object was shown for the first two seconds and then the object for the next trial appeared. The mean time taken to answer per question and the percentage of correct answers were measured.

(4) Rendering Virtual Objects

We used the isotropic Ward model as expressed in the following equation for object rendering.

$$
\begin{aligned}
& \rho_{b d}\left(\theta_{i}, \phi_{i}, \theta_{r}, \phi_{r}\right)= \\
& \frac{\rho_{d}}{\pi}+\rho_{s} \frac{1}{\sqrt{\cos \theta_{i} \cos \theta_{r}}} \frac{1}{4 \pi \alpha^{2}} \exp \left[-\tan ^{2}\left[\frac{\boldsymbol{h} \cdot \boldsymbol{n}}{\alpha^{2}}\right]\right]
\end{aligned}
$$

$\rho_{d}$ is the diffuse albedo, $\rho_{s}$ is the specular albedo, and $\alpha$ is the roughness. It is possible to express gloss and burnish of various materials by changing these three parameters.

We used three sets of parameters shown in Table 1 for virtual objects in reference to Ward's paper ${ }^{24)}$. The original parameters shown in Ward's paper ${ }^{24)}$ were the parameters of the anisotropic Ward model, but we modified them to the parameters of the isotropic Ward model to simplify the experiment. We determined the roughness parameter $\alpha$ of each material by choosing one of the two roughness parameters $\alpha_{x}$ and $\alpha_{y}$ that was easier to distinguish the difference.

The parameters for the common material faces were used to render the five faces with the same material and those for the different material face were used to render the other face with a different material. Figure 5 shows rendering examples of the objects used in the task.

To change the reflectance of the different material face of either Object A or B that was different from Reference, a variation was added to the roughness $\alpha$ of the face.

(5) Procedure

We conducted a within-participants experiment with
Table 1 Sets of parameters for virtual objects

\begin{tabular}{|c|c|ccc|}
\hline \multicolumn{2}{|c|}{ Parameter set } & $\rho_{d}$ & $\rho_{s}$ & $\alpha$ \\
\hline \hline \multirow{2}{*}{ I } & Common material faces & 0.15 & 0.19 & 0.088 \\
& Different material face & 0.1 & 0.33 & 0.16 \\
\hline \multirow{2}{*}{ II } & Common material faces & 0.7 & 0.05 & 0.071 \\
& Different material face & 0.45 & 0.059 & 0.08 \\
\hline \multirow{2}{*}{ III } & Common material faces & 0.45 & 0.043 & 0.13 \\
& Different material face & 0.67 & 0.07 & 0.092 \\
\hline
\end{tabular}
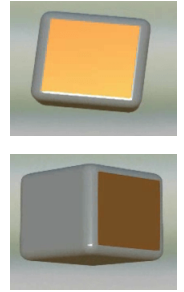

Parameter set I

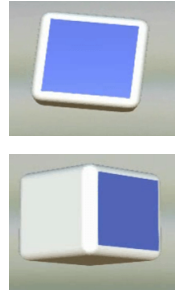

Parameter set II
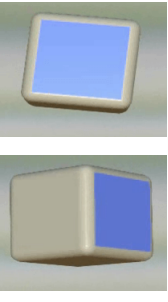

Parameter set III
Fig. 5 Rendering examples of the objects: (top) frontal view, (bottom) diagonal view

two independent variables (method, roughness variation). The method was either user-perspective or camera-perspective. The variation added to the roughness was $0.05,0.04,0.03,0.02$, or 0.01 , which was determined from the result of a preliminary experiment. We set the time limit per question to 90 seconds. Each participants did 2 methods $\times 5$ roughness variations $\times 3$ repetition $=30$ trials. Half of the participants performed the task with user-perspective first and the other participants performed the task with cameraperspective first.

The experiment took about two hours per participant. First, we explained the overview of the experiment to each participant, and asked demographic questions. Then, the participant performed the task with each method in the following steps. Ten minutes break was given between the methods.

(1) explanation and demonstration of the method

(2) practice with the method (10 to 15 minutes)

(3) performing the task (15 questions, 1 minutes break per five questions)

(4) answering the questionnaire for the method

When the participants were manipulating a virtual object using our system, there was no physical object between their fingers, and there was concern that they could pinch their fingers too much and penetrate the virtual object. Therefore, when the participants were practicing using the system, we explained them to grasp an object without their fingers getting into the object, and confirmed that they were able to do so.

In the questionnaire, we used Raw-TLX ${ }^{25)}$ to measure 


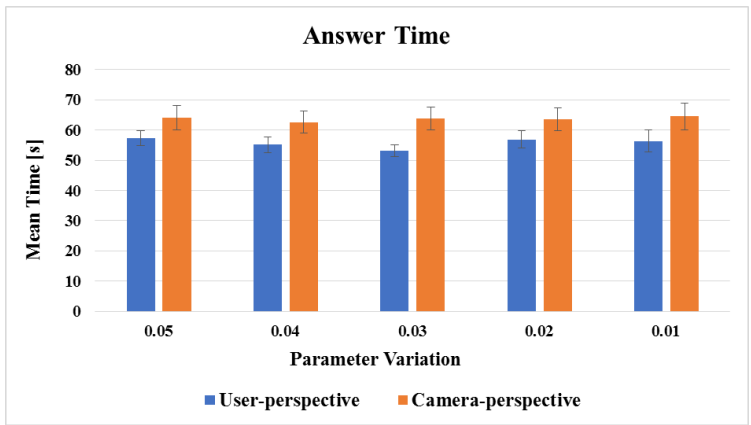

Fig. 6 Mean answer time (error bars indicate standard deviations)

the load of the task with each method. In addition, we asked several questions such as efficiency to use, easiness to use, easiness to learn, feeling of reality, and feeling of touch with a 5-point Likert scale.

We asked the participants to answer a question soon when they found the answer. After finishing the task with all the methods, we asked them to give feedback to the experiment.

\section{3 Results}

Figure 6 shows mean answer time with different roughness variation. The ANOVA indicated a significant main effect of method $(F(1,11)=13.86, p=$ .00336) but no significant main effect of roughness variation $(F(4,44)=0.34, p=.847)$ or interaction between method and roughness variation $(F(4,44)=$ $0.21, p=.933)$. This result shows that user-perspective enabled significantly shorter answer time than cameraperspective.

It took about one minute on average for the participants to answer. Most of the participants switched the object twice for one trial, which means they checked each object once and did not check the same objects repeatedly. They checked each object for almost the same amount of time. Therefore, it took twenty minutes to check each object.

Figure 7 shows mean correct answer rate with different roughness variation. The ANOVA indicated no significant main effect of method $(F(1,11)=1.45, p=$ $.254)$ or roughness variation $(F(4,44)=1.25, p=.303)$, or interaction between method and roughness variation $(F(4,44)=0.21, p=.933)$. This result shows there were no significant difference in correct answer rates between user-perspective and camera-perspective.

The mean score of the NASA-TLX with userperspective was slightly lower (better) than that with camera-perspective, but the Wilcoxon signed-rank test indicated no significant difference in the scores between

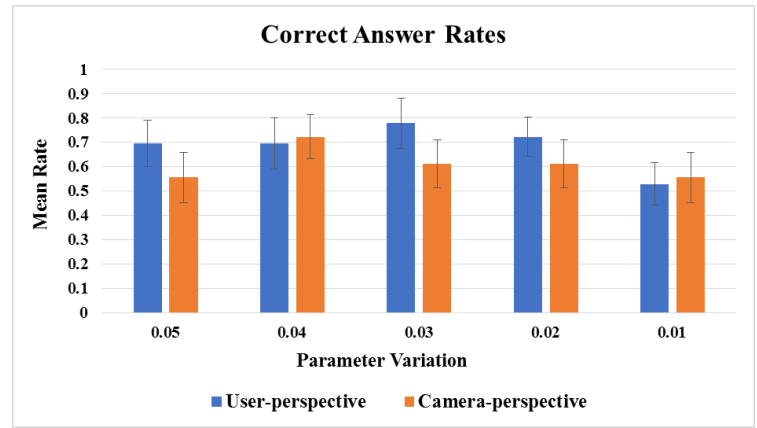

Fig. 7 Mean correct answer rates (error bars indicate standard deviations)

user-perspective and camera-perspective. On the other hand, the Wilcoxon signed-rank test for each item of the NASA-TLX indicated that there were significant difference in frustration $(V=11.5, p=.0283)$. This result shows the participants felt significantly less stress or discomfort with user-perspective than with cameraperspective.

About other questions in the questionnaire, though user-perspective gained higher (better) mean scores for all the items than camera-perspective, the Wilcoxon signed-rank test showed a significant difference only in feeling of reality $(V=28.0, p=.0156)$. This result shows that the participants felt a greater sense of reality in manipulating objects with user-perspective.

Below are feedback comments we received from the participants. Since the participants did not know the names of the methods, they answered like "The first method was ... than the second method", but we replaced the words by the original names of the methods.

- I felt that I was able to perform the task better with user-perspective than camera-perspective.

- I was able to use the system more intuitively and felt less stress with user-perspective than cameraperspective.

- I was able to focus more on the gloss with cameraperspective than user-perspective.

- I could not tell the difference between userperspective and camera-perspective.

- It was fun to manipulate objects with my hand. I felt like I were actually touching the objects.

- It was difficult to rotate objects around the vertical axis.

- My thumb hurt when I manipulated objects with my hand. 


\section{Discussion}

The result of experiment showed that userperspective mobile AR gave shorter answer time, less stress or discomfort, and a greater sense of reality in the object manipulation task for material perception than camera-perspective mobile AR. When manipulating virtual objects with the user's hand, the users' visual and somatosensory perception became consistent with the user-perspective presentation, which may have improved the usability of the system and reduced the time taken for operation.

Meanwhile, there was no significant difference in the answer rates and we cannot say that there is any effect on material perception. The system we used in the experiment has a problem of manipulation accuracy, which was also mentioned in the participants' comments. This problem might have made a bad influence on material perception with both modes, and have made the difference unclear. Another possible reason is that the questions were too difficult to answer correctly. We determined the variations added to the roughness parameter using the result of the preliminary experiment, but the result showed that the correct answer rates were still low with both perspective modes, which made the difference between the perspective modes small.

Besides our study, there are some other studies that compared user and camera (or device) perspectives. Baricevic et al. evaluated the benefits of userperspective presentation in AR with mobile devices ${ }^{13)}$. Their experiment was conducted in a simulated environment using their VR system. Instead of direct hand interaction, the participants controlled a blue pointer with a wand and its position was displayed on the screen. They measured the time to select the target with the pointer after it enters the field of view, and the error in the path traveled by the pointer. There was no significant difference in selection time between perspectives, but path deviation was a little shorter in user-perspective presentation. Pucihar et al. analyzed users' expectations and spatial perception by comparing user and device perspectives on a commercially available mobile device ${ }^{20)}$. The participants performed a task in which they specified a position in real space that corresponded to the position displayed on the mobile device by touching a large touchscreen below the device. The majority of participants expected userperspective presentation and also demonstrated signif- icantly better spatial perception and preference of the user-perspective view. Lima et al. compared user and device perspectives using Google Glass ${ }^{26)}$. The participants were asked to position magnetic polygonal pieces on a vertical metal surface at the locations highlighted by an AR application. The result showed that the participants achieved better precision with deviceperspective presentation and also preferred it.

While all of these studies made comparisons based on a positioning task, our study used a more complicated task with 6-DOF object manipulation, and we were able to show significant difference in task completion time. In addition, although we could not show significant difference, this is the first study to examine the benefit of user-perspective presentation on material perception.

\section{Conclusion}

In this study, we conducted an experiment using our system to evaluate the effectiveness of user-perspective presentation in mobile AR. We compared the methods with user-perspective and camera-perspective presentations using an object manipulation task for material perception. The result showed that the answer time in the task became significantly shorter with userperspective presentation. Though no significant difference were seen in the answer rates, but there is a possibility of improving the perception of materials by solving the problem of manipulation accuracy in our system.

\section{References}

1) Maria Luz Caballero, Ting-Ray Chang, Maria Menendez, and Valentina Occhialini. Behand: Augmented virtuality gestural interaction for mobile phones. In Proceedings of the 12th International Conference on Human Computer Interaction with Mobile Devices and Services, pp. 451-454, 2010.

2) Matthias Baldauf, Sebastian Zambanini, Peter Fröhlich, and Peter Reichl. Markerless visual fingertip detection for natural mobile device interaction. In Proceedings of the 13th International Conference on Human Computer Interaction with Mobile Devices and Services, pp. 539-544, 2011.

3) Wolfgang Hurst and Casper Van Wezel. Gesture-based interaction via finger tracking for mobile augmented reality. Multimedia Tools and Applications, Vol. 62, No. 1, pp. 233-258, 2013.

4) Wendy H. Chun and Tobias Hollerer. Real-time hand interaction for augmented reality on mobile phones. In Proceedings of the 2013 International Conference on Intelligent User Interfaces, pp. 307-314, 2013.

5) Huidong Bai, Lei Gao, Jihad El-Sana, and Mark Billinghurst. Markerless 3d gesture-based interaction for handheld augmented reality interfaces. In Proceedings of the 12th IEEE International Symposium on Mixed and Augmented Reality, pp. 1-6, 2013.

6) Huidong Bai, Gun A. Lee, Ramakrishnan Mukundan, and Mark Billinghurst. $3 \mathrm{~d}$ gesture interaction for handheld augmented reality. In Proceedings of the SIGGRAPH Asia 2014 Mobile Graphics and Interactive Applications, pp. 7:1-7:6, 2014.

7) Minseok Kim and Jae Yeol Lee. Touch and hand gesture-based interactions for directly manipulating $3 \mathrm{~d}$ virtual objects in mobile augmented reality. Multimedia Tools and Applications, Vol. 75, No. 23, pp. 16529-16550, 2016. 
8) Brandon Yee, Yuan Ning, and Hod Lipson. Augmented reality in-situ 3d sketching of physical objects. In Proceedings of the 2009 International Conference on Intelligent User Interfaces Workshop on Sketch Recognition, 2009.

9) Michael Csongei, Liem Hoang, Ulrich Eck, and Christian Sandor. Clonar: Rapid redesign of real-world objects. In Proceedings of the 11th IEEE International Symposium on Mixed and Augmented Reality, pp. 277-278, 2012.

10) Ya-Ting Yue, Xiaolong Zhang, Yongliang Yang, Gang Ren, YiKing Choi, and Wenping Wang. Wiredraw: 3d wire sculpturing guided with mixed reality. In Proceedings of the 2017 CHI Conference on Human Factors in Computing Systems, pp. 36933704, 2017.

11) Anuruddha Hettiarachchi and Daniel Wigdor. Annexing reality: Enabling opportunistic use of everyday objects as tangible proxies in augmented reality. In Proceedings of the 2016 CHI Conference on Human Factors in Computing Systems, pp. 1957-1967, 2016.

12) Yuki Ban, Takuji Narumi, Tatsuya Fujii, Sho Sakurai, Jun Imura, Tomohiro Tanikawa, and Michitaka Hirose. Augmented endurance: Controlling fatigue while handling objects by affecting weight perception using augmented reality. In Proceedings of the 2013 CHI Conference on Human Factors in Computing Systems, pp. 69-78, 2013.

13) Domagoj Baricevic, Cha Lee, Matthew Turk, Tobias Hollerer, and Doug A Bowman. A hand-held ar magic lens with userperspective rendering. In Proceedings of the 11th IEEE International Symposium on Mixed and Augmented Reality, pp. 197206, 2012.

14) Domagoj Baricevic, Tobias Hollerer, Pradeep Sen, and Matthew Turk. User-perspective augmented reality magic lens from gradients. In Proceedings of the 20th ACM Symposium on Virtual Reality Software and Technology, pp. 87-96, 2014.

15) Makoto Tomioka, Sei Ikeda, and Kosuke Sato. Approximated user-perspective rendering in tablet-based augmented reality. In Proceedings of the 12th IEEE International Symposium on Mixed and Augmented Reality, pp. 21-28, 2013.

16) Daniel Andersen, Voicu Popescu, Chengyuan Lin, Maria Eugenia Cabrera, Aditya Shanghavi, and Juan Wachs. A hand-held, selfcontained simulated transparent display. In Proceedings of the 15th IEEE International Symposium on Mixed and Augmented Reality, pp. 96-101, 2016.

17) Peter Mohr, Markus Tatzgern, Jens Grubert, Dieter Schmalstieg, and Denis Kalkofen. Adaptive user perspective rendering for handheld augmented reality. In Proceedings of the 2017 IEEE Symposium on 3D User Interfaces, pp. 176-181, 2017.

18) Thomas Schops, Martin R. Oswald, Pablo Speciale, Shuoran Yang, and Marc Pollefeys. Real-time view correction for mobile devices. IEEE Transactions on Visualization and Computer Graphics, Vol. 23, pp. 2455-2462, 2017.

19) Yuko Unuma and Takashi Komuro. Natural 3d interaction using a see-through mobile ar system. In Proceedings of the 14 th IEEE International Symposium on Mixed and Augmented Reality, pp. 84-87, 2015.

20) Klen Copic Pucihar, Paul Coulton, and Jason Alexander. Evaluating dual-view perceptual issues in handheld augmented reality: Device vs. user perspective rendering. In Proceedings of the 15th ACM International Conference on Multimodal Interaction, pp. 381-388, 2013.

21) Klen Copic Pucihar, Paul Coulton, and Jason Alexander. The use of surrounding visual context in handheld ar: device vs. user perspective rendering. In Proceedings of the SIGCHI Conference on Human Factors in Computing Systems, pp. 197-206, 2014.

22) Ricardo A. Borsoi and Guilherme H. Costa. On the performance and implementation of parallax free video see-through displays. IEEE Transactions on Visualization and Computer Graphics, Vol. 24, No. 6, pp. 2011-2022, 2018.

23) Ryota Nomura, Yuko Unuma, Takashi Komuro, Shoji Yamamoto, and Norimichi Tsumura. Mobile augmented reality for providing perception of materials. In Proceedings of the 16th International Conference on Mobile and Ubiquitous Multimedia, pp. 501-506, 2017.

24) Gregory J. Ward. Measuring and modeling anisotropic reflection. In Proceedings of the 19th Annual Conference on Computer Graphics and Interactive Techniques, pp. 265-272, 1992.

25) Sandra G. Hart. Nasa-task load index (nasa-tlx); 20 years later. In Proceedings of the human factors and ergonomics society annual meeting, Vol. 50, pp. 904-908, 2006.

26) Joao P. Lima, Rafael Roberto, Joao Teixeira, and Veronica Teichrieb. Device vs. user perspective rendering in google glass ar applications. In Proceedings of 2014 IEEE International Symposium on Mixed and Augmented Reality, pp. 279-280, 2014.

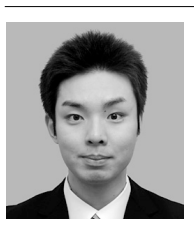

Ryota Nomura received the B.E. and M.E. degrees in information and computer sciences from Saitama University in 2017 and 2019, respectively. At present he works for Softbank Corp.

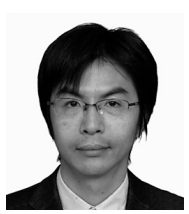

Takashi Komuro received the B.E., M.E. and $\mathrm{Ph} . \mathrm{D}$. degrees in mathematical engineering and information physics from the University of Tokyo in 1996, 1998, and 2001, respectively. At present he is a professor of mathematics, electronics and informatics at Saitama University. His current research interests include image sensing, computer vision, user interfaces, and virtual/augmented reality.

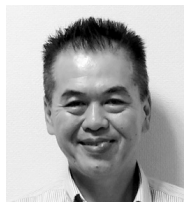

Shoji Yamamoto received his B.E. and M.E. degrees from the Department of Opt-ElectroMechanics Engineering from Shizuoka University in 1989 and 1991 respectively. And he received Dr. Eng degrees in information science from Chiba University in 2007. He is currently Professor in Tokyo Metropolitan College of Industrial Technology, Japan. He is interested in Vision science, Image processing, and Computer graphics. He is a member of the Optical Society of Japan, the Institute of Image Information and Television Engineers.

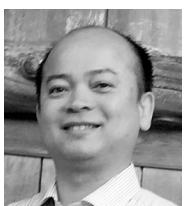

Norimichi Tsumura received his B.E.

M.E., and Dr. Eng. degrees in Applied Physics from Osaka University in 1990, 1992, and 1995 , respectively. He is currently an associate professor in the Department of Information and Image Sciences, Chiba University (since February 2002). 